\title{
The effect of proton pump inhibitor uses on outcomes for cancer patients treated with immune checkpoint inhibitors: a meta- analysis
}

\author{
Chao Li", Zhengzheng Xia", Anna Li, Jun Meng \\ National Cancer Center/National Clinical Research Center for Cancer/Cancer Hospital \& Shenzhen Hospital, Chinese Academy of Medical \\ Sciences and Peking Union Medical College, Guangdong, Shenzhen, China \\ Contributions: (I) Conception and design: C Li; (II) Administrative support: J Meng; (III) Provision of study materials or patients: A Li, Z Xia, J Meng; \\ (IV) Collection and assembly of data: Z Xia, A Li; (V) Data analysis and interpretation: C Li, Z Xia; (VI) Manuscript writing: Z Xia; (VII) Final \\ approval of manuscript: All authors. \\ \#These authors contributed equally to this work. \\ Correspondence to: Jun Meng. Department of Pharmacy, Cancer Hospital \& Shenzhen Hospital, Chinese Academy of Medical Sciences and Peking \\ Union Medical College, Baohe Main Street 113, Longgang District, Shenzhen 518116, China. Email: 497963464@qq.com.
}

\begin{abstract}
Background: Proton pump inhibitors (PPIs) are commonly prescribed for preventing and treating gastrointestinal ulcers. In patients with advanced cancer, it is unclear whether concomitant use of immune checkpoint inhibitors (ICIs) and PPIs could result in poorer outcomes. This study investigates the impact of PPIs on the survival of cancer patients treated with ICIs.

Methods: PubMed, MEDLINE, EMBASE, and the Cochrane Library databases were searched from January 1, 1970 to June 2, 2020 for studies reporting the prognoses of cancer patients receiving antitumor therapies including ICIs with or without PPIs. The primary and secondary endpoints were overall survival (OS) and progression-free survival (PFS), respectively. Hazard ratios (HRs) with a 95\% confidence interval (CI) were reported to express the effectiveness of ICIs in PPI users compared to non-PPI users, using a random effects model.
\end{abstract}

Results: Five studies were identified comprising 1,167 cancer patients. Concomitant use of PPIs with ICIs did not result in statistically significant changes in OS (HR 0.996; 95\% CI: 0.486-1.447) and PFS (HR 0.858 ; $95 \%$ CI: $0.388-1.328$ ). Statistical testing suggested heterogeneity among studies. Sensitivity analyses confirmed the stability of our results.

Conclusions: Concomitant ICI-PPI therapy does not appear to be significantly associated with OS or PFS, and further research on the effect of individual ICIs in different cancer patients is needed.

Keywords: Cancer; immune checkpoint inhibitor (ICI); survival; proton pump inhibitor (PPI); meta-analysis

Submitted Oct 16, 2020. Accepted for publication Dec 13, 2020.

doi: $10.21037 /$ atm-20-7498

View this article at: http://dx.doi.org/10.21037/atm-20-7498

\section{Introduction}

In the past decade, immune checkpoint inhibitors (ICIs) including cytotoxic $\mathrm{T}$ lymphocyte-associated antigen 4 (CTLA-4) inhibitors, programmed cell death-1 (PD-1) inhibitors, and programmed cell death-ligand 1 (PD-L1) inhibitors, have shown breakthrough efficacy in the management of a number of advanced malignancies (1).

Evidence has shown that the gut microbiome is related to the response to ICI immunotherapy (2-5). For example, concomitant use of broad-spectrum antibiotics is increasingly considered to play an important role in ICI 
therapeutic outcomes, as the diversity and abundance of the gut microbiome are associated with broad spectrum antibiotics $(6,7)$.

Proton pump inhibitors (PPIs), acting through the irreversible inhibition of the hydrogen/potassium pump (H+/K+-ATPase pump) in the gastric parietal cells, could change the $\mathrm{pH}$ value of the gut, thus causing changes to the gut microbiome.

Recent studies have shown a variable influence of PPIs on the efficacy of ICIs, ranging from no difference to a negative impact (8-14). Currently, the role of concomitant PPI use on ICIs' therapeutic efficacy remains controversial, thus a meta-analysis may provide a better understanding of this issue. To our knowledge, this is the first meta-analysis to systematically evaluate the association between PPI use and the clinical efficacy of ICIs. Assessing the impact on the clinical efficacy of adding PPI use to ICI immunotherapy would help to establish therapeutic decision-making algorithm among cancer patients who were also potential candidates for PPIs. We present the following article in accordance with the PRISMA reporting checklist (available at http://dx.doi.org/10.21037/atm-20-7498).

\section{Methods}

\section{Registration}

This study was registered in PROSPERO with registration number CRD42020190094.

\section{Retrieval of studies}

This meta-analysis was performed in accordance with the Preferred Reporting Items for Systematic Reviews and Meta-Analyses (PRISMA) statement (15).

The search was performed from January 1, 1970 to June 2, 2020 and included studies written in English in PubMed, MEDLINE, EMBASE, and the Cochrane Library databases using the terms (PPI or proton pump inhibitor) AND (PD-1 or PD-L1 or CTLA-4 or "immune checkpoint inhibitors") AND survival (Appendix 1).

Studies that satisfied all of the following criteria were included: (I) patients: patients were diagnosed with cancer, and had received antitumor therapy including ICIs alone (CTLA-4 inhibitors and PD-1/L1 inhibitors) or in combination; (II) intervention: PPIs were prescribed before, during, or after the antitumor therapeutic course; (III) comparison: the experimental group received PPIs but the control group did not; (IV) outcome: complete outcomes that measured overall survival (OS) and progression-free survival (PFS) were extracted.

Initially, citations containing the key terms of the search were evaluated by their titles and abstracts. Subsequently, full texts of relevant citations were further assessed for possible inclusion in the systematic review. Conflicts amongst the researchers regarding study selection were resolved by discussion, referring back to the original article until consensus was achieved between all authors.

\section{Quality evaluation}

The Newcastle-Ottawa quality assessment scale (NOS) was adopted to evaluate the quality of each study on these items: patient selection, comparability of groups, and assessment of outcome (16). Studies with less than six stars were regarded as relatively low quality and were excluded. Two investigators independently evaluated the risk of bias, and disagreements were resolved through discussion until consensus was achieved.

\section{Data extraction}

Two independent investigators separately screened the titles and abstracts of eligible studies by applying the inclusion criteria, and any discrepancies between them were resolved by consensus between all authors. Information was extracted from the eligible articles as follows (where available): authors, year of publication, country, title, number of patients, type of cancer, type of ICIs and PPIs, PFS, OS, and hazard ratios (HRs). Missing data was handled by contacting the authors of the studies for unreported data or additional details.

\section{Data analysis}

The primary and secondary outcomes were OS and PFS, respectively. HRs with a $95 \%$ confidence interval (CI) were calculated to express the effectiveness of ICIs in PPI users compared to non-PPI users. Heterogeneity among the studies was evaluated by $\mathrm{I}^{2}$ statistics. When $\mathrm{I}^{2}>50 \%$ and/ or $\mathrm{P}<0.10$, the heterogeneity was considered statistically significant. Under this circumstance, a random effects model was adopted to pool the HRs; otherwise, a fixed effects model was adopted (17).

Publication bias assessment was not performed due to 

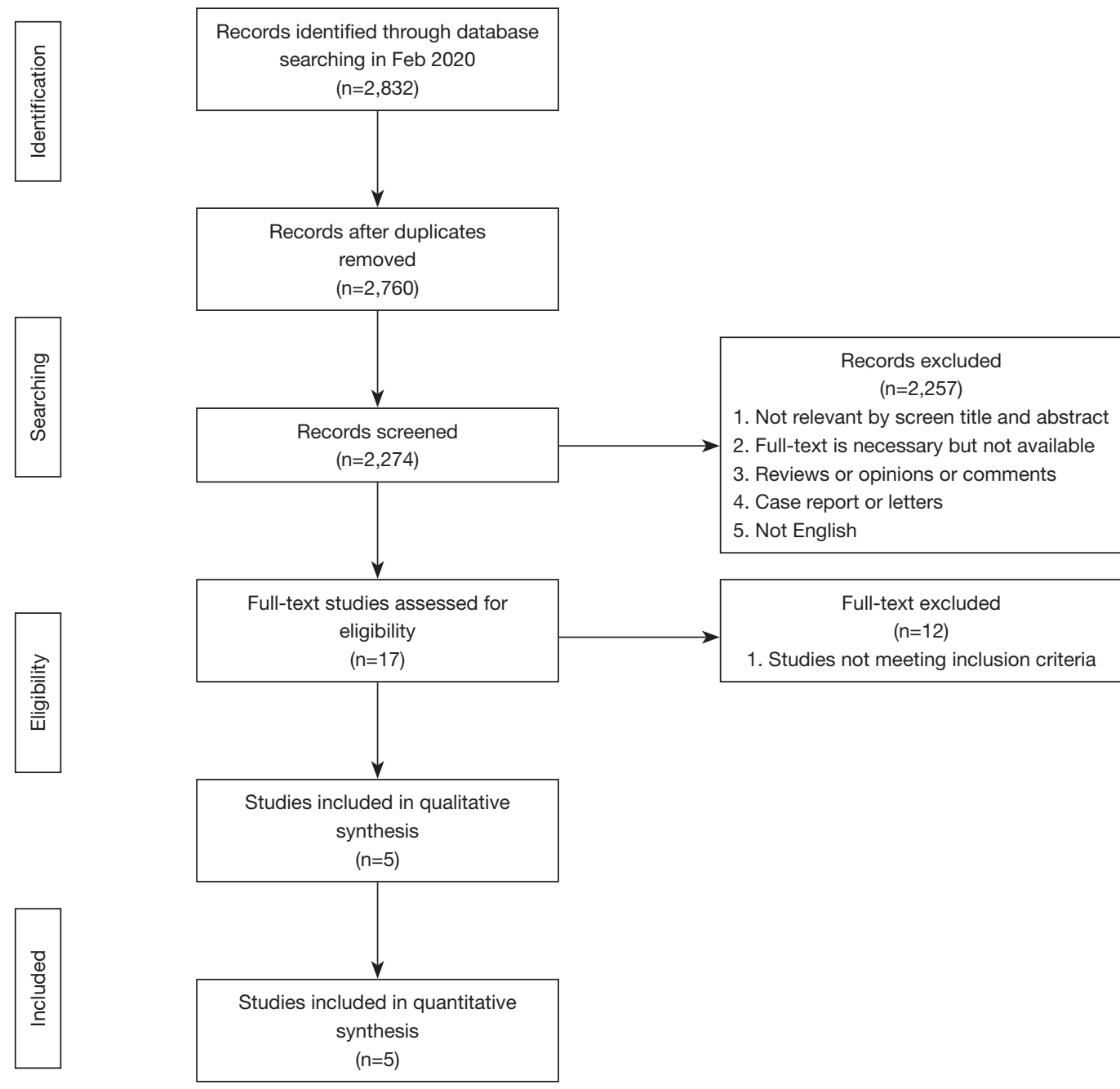

Figure 1 Flowchart of study selection procedure.

the limited number of studies (below 10) included in each analysis. Sensitivity analyses were performed by individual exclusion of each study for each outcome to assess their effect on the pooled outcome hazard ratio. All statistical analyses were conducted on Stata software (Version 15.0, Stata Corporation, College Station, TX, USA).

\section{Results}

\section{Search results}

A total of 1,167 patients from five studies were eligible for quantitative analysis (18-22) (Figure 1).

The basic characteristics of the enrolled studies are shown in Table 1. All of the studies were retrospective. Among them, four studies were complete cohort studies and one study provided only an abstract. The included studies were published between 2016 and 2020 (with four of the studies published in 2019 and 2020) from China, the United States, Spain, and Switzerland/Netherlands/USA.

PPIs were given to $52.0 \%$ of patients. In regard to the time of PPI use, two studies reported patients taking PPIs at the time of ICI initiation, two studies reported patients taking PPIs within 1 month before or after the first dose of ICIs, and one study did not report the time window of PPI use. In regard to ICI type, two studies included antiCTLA-4 inhibitors and/or anti-PD-1/L1 inhibitors, one 


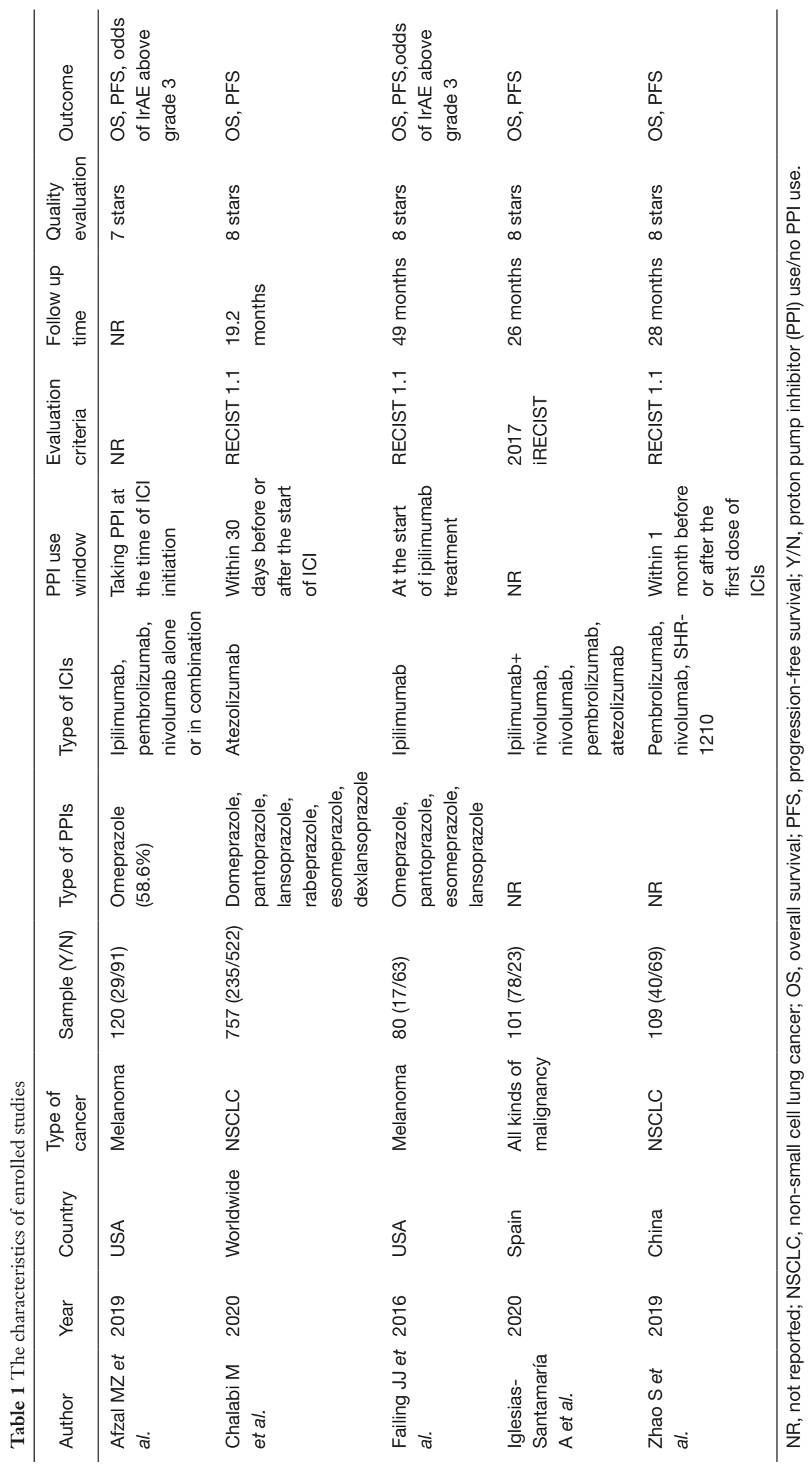


study included anti-CTLA-4 inhibitors alone, one study used anti-PD-1 inhibitors alone, and a further study included anti-PD-L1 inhibitors alone.

\section{Main results}

OS was not significantly associated with the concomitant use of PPIs (HR $=0.966,95 \%$ CI: 0.486-1.447; Figure $2 A)$. Owing to high heterogeneity $\left(\mathrm{I}^{2}=74.3 \%\right.$ ), a random effects model was used. The subgroup analyses were performed based on the type of cancer and the type of ICI drug. (NSCLC: $\mathrm{HR}=1.103,95 \%$ CI: $0.353-1.854, \mathrm{P}=0.014$, $\mathrm{I}^{2}=83.4 \%$; non-NSCLC: $\mathrm{HR}=0.788,95 \%$ CI: $0.277-1.298$, $\left.\mathrm{P}=0.212, \mathrm{I}^{2}=35.5 \%\right)$. The subgroup analyses based on the type of ICI drug obtained the same results.

PFS was not significantly associated with the concomitant use of PPIs (HR $=0.858,95 \%$ CI: $0.388-1.328$; Figure $2 B$ ). Owing to high heterogeneity $\left(\mathrm{I}^{2}=87.7 \%\right)$, a random effects model was used. The subgroup analyses were performed based on the type of cancer and the type of ICI drug. (NSCLC: $\mathrm{HR}=1.173,95 \%$ CI: $0.815-1.521, \mathrm{P}=0.160$, $I^{2}=49.3 \%$; non-NSCLC: $\mathrm{HR}=0.680,95 \%$ CI: $0.173-1.042$, $\left.\mathrm{P}=0.050, \mathrm{I}^{2}=66.6 \%\right)$. The sensitivity analyses revealed that the results were not dominated by any single study (Figure $3 A, B$ ). Subgroup analyses based on the type of ICI drug obtained the same results.

Subgroup analyses of the effect of types of PPI or the timing and course of PPIs were not possible due to incomplete data.

Publication bias assessment was not performed due to the limited number of studies (below 10) included in each analysis.

\section{Discussion}

Previous evidence has shown that an intact gut microbiota is necessary in order to create the immune response and provide ICI treatment benefits to cancer patients $(23,24)$. Unlike previous studies, this study focused on the effect of the interaction between PPIs and ICIs. Our conclusion is that use of PPIs does not appear to affect the clinical efficacy of ICIs. This result is contrary to previous theories suggesting that antibiotic use which alters the microbiota is associated with a poorer response to immunotherapy.

PPI-driven gastric hypochlorhydria can cause significant changes in the gut microbiota composition, and PPI use was therefore reported to be associated with decreased bacterial richness, gut dysbiosis, and the promotion of T-cell tolerance. Hypochlorhydria also promotes a reduction in microbial diversity and the growth of microbes that have genotoxic potential, with an increase in the nitrate/ nitrite reductase bacterial functions involved in cancer development (25-27). The effect of PPIs is not only limited to influencing gut microbiota but can also affect tumor growth. It has been reported that PPIs play a role in regulating the acidic microenvironment of tumor cells and in facilitating the proliferation, progression, and metastasis of tumors $(28,29)$. PPIs also sensitize the resistant tumors to chemotherapeutic and immunotherapeutic effects $(30,31)$. Thus, it is hard to predict the net result of the changes caused by PPIs for cancer patients who are on ICI immunotherapy.

Assessing the impact on the clinical efficacy of adding PPI use to ICI immunotherapy would help to establish a therapeutic decision-making algorithm for physicians treating cancer patients who are also potential candidates for PPIs.

The limitations of this meta-analysis were as follows: Firstly, it consisted of retrospective studies with heterogeneous populations with a diversity of cancer types, stages, and patient characteristics. Secondly, the type of PPI, indication, time frame and duration of PPIs were not available by search. Finally, antitumor agents other than ICIs as well as other concomitant drugs other than PPIs used for patients were not taken into consideration.

Therefore, further prospective studies, especially of patients with esophageal cancer or gastrointestinal cancer, are necessary to identify which specific phenotypes of gut microbiota may strengthen or weaken antitumor immune responses as a result of the use of PPIs. Validation of the evaluation results of co-administration of PPIs and ICIs from completed or ongoing randomized controlled trials is needed, taking into account that prospective trials of PPIs on outcome after ICI may be impracticable.

\section{Conclusions}

In summary, the current meta-analysis evaluating the aforementioned studies did not suggest that the concomitant use of PPIs and ICIs in cancer patients was associated with a significant difference in OS or PFS. Clinical trials to investigate the modulating mechanism of the gut microbiome in order to enhance ICI efficacy should be actively conducted. 
A Study

ID

ES $(95 \% \mathrm{Cl}) \quad$ Weight

Afzal, M. Z. (2019)
Chalabi, M. (2020)
Failing, Jarrett J. (2016)
Iglesias-Santamaria, A (2020)
Zhao, S. (2019)
Overall (I-squared $=74.3 \%, \mathrm{p}=0.004)$
Nore: Waights are trom random affects analysis

B

Study

ID
ES $(95 \% \mathrm{Cl}) \quad$ Weight

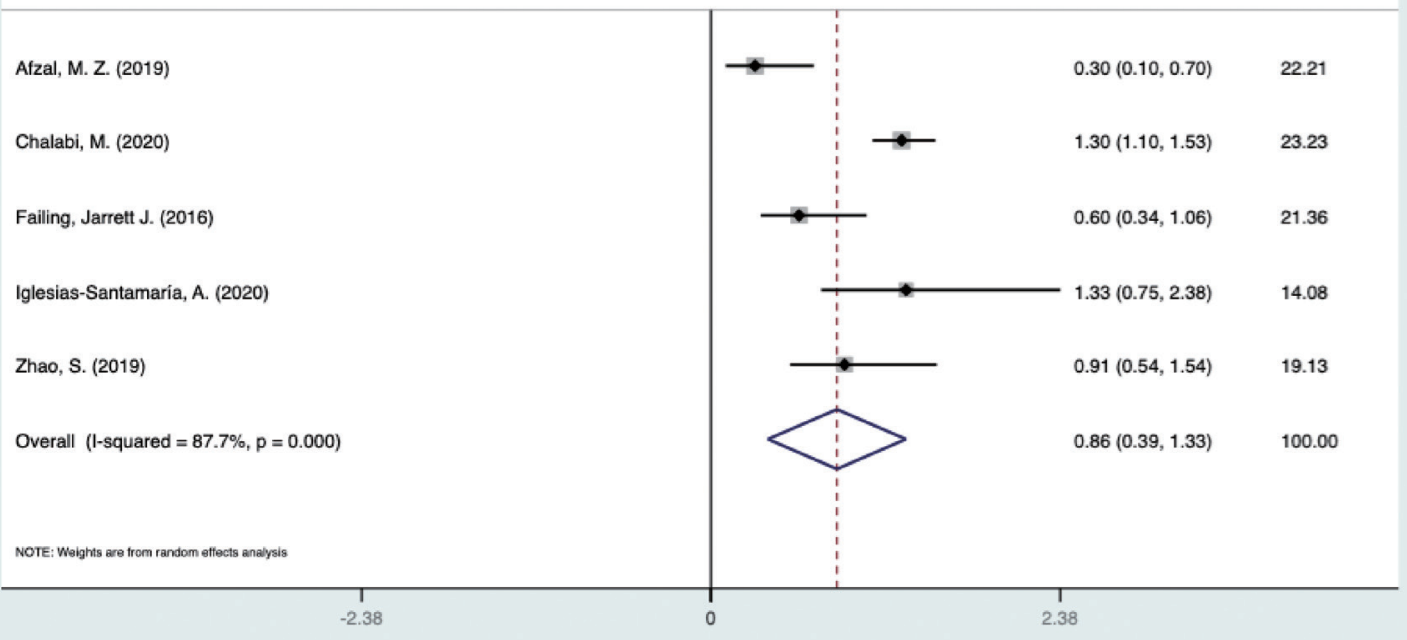

Figure 2 The associations between PPI use and overall survival (A) and progression-free survival (B) in cancer patients treated with ICI. PPI, proton pump inhibitor; ICI, immune checkpoint inhibitor. 
A

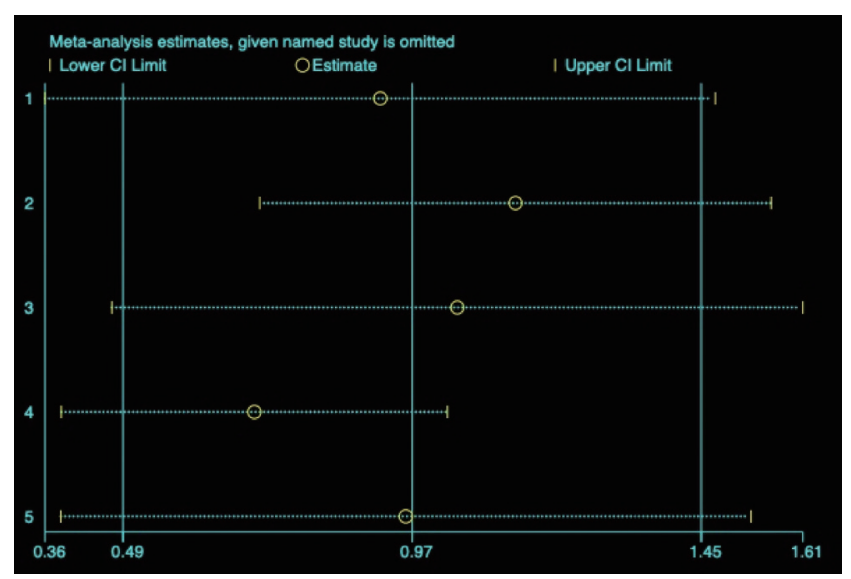

B

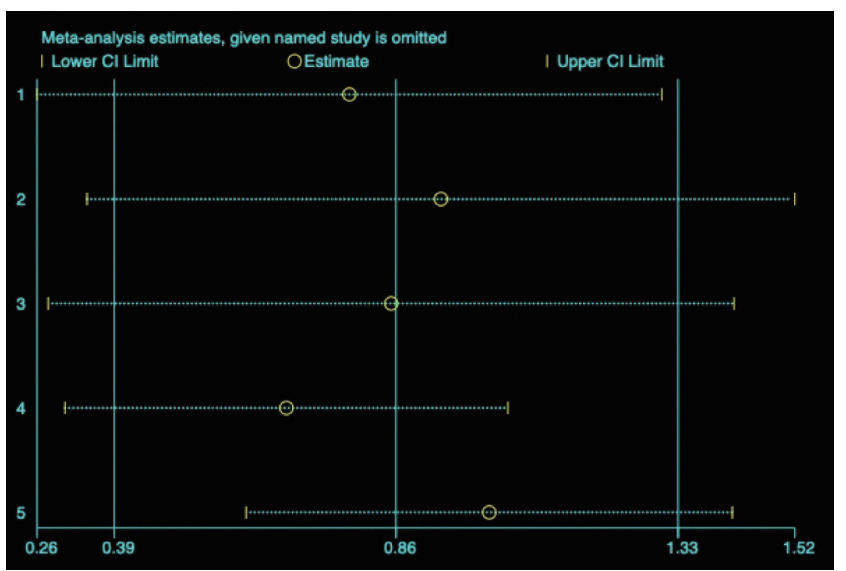

Figure 3 Sensitivity analysis of overall survival and (A) progression-free survival (B).

\section{Acknowledgments}

Funding: This research is supported by Shenzhen Science and technology project No. JCY20170307112807219.

\section{Footnote}

Reporting Checklist: The authors have completed the PRISMA reporting checklist. Available at http://dx.doi. org/10.21037/atm-20-7498

Conflicts of Interest: All authors have completed the ICMJE uniform disclosure form (available at http://dx.doi. org/10.21037/atm-20-7498). The authors have no conflicts of interest to declare.

Ethical Statement: The authors are accountable for all aspects of the work in ensuring that questions related to the accuracy or integrity of any part of the work are appropriately investigated and resolved.

Open Access Statement: This is an Open Access article distributed in accordance with the Creative Commons Attribution-NonCommercial-NoDerivs 4.0 International License (CC BY-NC-ND 4.0), which permits the noncommercial replication and distribution of the article with the strict proviso that no changes or edits are made and the original work is properly cited (including links to both the formal publication through the relevant DOI and the license). See: https://creativecommons.org/licenses/by-nc-nd/4.0/.

\section{References}

1. Ribas A, Wolchok JD. Cancer immunotherapy using checkpoint blockade. Science 2018;359:1350-5.

2. Roy S, Trinchieri G. Microbiota: a key orchestrator of cancer therapy. Nat Rev Cancer 2017;17:271-85.

3. Routy B, Chatelier EL, Derosa L, et al. Gut microbiome influences efficacy of PD-1-based immunotherapy against epithelial tumors. Science 2018;359:91-7.

4. Gori S, Inno A, Belluomini L, et al. Gut microbiota and cancer: How gut microbiota modulates activity, efficacy and toxicity of antitumoral therapy. Crit Rev Oncol Hematol 2019;143:139-47.

5. Havel JJ, Chowell D, Chan IA. The evolving landscape of biomarkers for checkpoint inhibitor immunotherapy. Nat Rev Cancer 2019;19:133-50.

6. Huang X, Gao P, Song Y, et al. Antibiotic use and the efficacy of immune checkpoint inhibitors in cancer patients: a pooled analysis of 2740 cancer patients. Oncoimmunology 2019;8:e1665973.

7. $\mathrm{Xu} \mathrm{H}, \mathrm{Xu} \mathrm{X}$, Wang $\mathrm{H}$, et al. The association between antibiotics use and outcome of cancer patients treated with immune checkpoint inhibitors: A systematic review and meta-analysis. Crit Rev Oncol Hematol 2020;149:102909.

8. Homicsko K, Richtig G, Tuchmann F, et al. Proton pump inhibitors negatively impact survival of PD-1 inhibitor based therapies in metastatic melanoma patients. Ann Oncol 2018;29:x40.

9. Izawa N, Shiokawa H, Onuki R, et al. Association of gastric acid suppression with efficacy of immune 
checkpoint inhibitors (ICIs) in advanced cancer patients. Ann Oncol 2019;30:vi115.

10. Kulkarni A, Kumar M, Pease DF, et al. Impact of antibiotics and proton pump inhibitors on clinical outcomes of immune check point blockers in advanced non-small cell lung cancers and metastatic renal cell cancer. J Clin Oncol 2019;37:abstr e20520.

11. Nguyen QP, Nomura M, Matsumoto S, et al. PPI the effect of proton pump inhibitors on the efficacy of nivolumab monotherapy in different types of cancer. Ann Oncol 2019;30:vi115.

12. Rossi G, Pezzuto A, Sini C, et al. Concomitant medications during immune checkpoint blockage in cancer patients: Novel insights in this emerging clinical scenario. Crit Rev Oncol Hematol 2019;142:26-34.

13. Spakowicz D, Husain M, Tinoco G, et al. Effect of concomitant medications on overall survival in patients with cancer undergoing immunotherapy. J Clin Oncol 2019;37:94.

14. Trabolsi A, Winter M, Rodriguez E. Proton pump inhibitors and response to immune check-point inhibitors: Single center study. J Clin Oncol 2019;37:abstr e14092.

15. Moher D, Liberati A, Tetzlaff J, et al. Preferred reporting items for systematic reviews and meta-analyses: the PRISMA statement. BMJ 2009;339:b2535.

16. Wells GA, Shea B, O'Connell D, et al. The NewcastleOttawa Scale (NOS) for Assessing The Quality of Nonrandomised Studies in Meta-analyses. Available online: http://wwwohrica/programs/clinical_epidemiology/ oxfordasp. 2020-10-06 2020.

17. Higgins JPT, Thompson SG, Deeks JJ, et al. Measuring inconsistency in meta-analyses. BMJ 2003;327:557-60.

18. Failing JJ, Finnes HD, Kottschade LA, et al. Effects of commonly used chronic medications on the outcomes of ipilimumab therapy in patients with metastatic melanoma. Melanoma Res 2016;26:609-15.

19. Afzal $M Z$, Shirai K. What impact do the proton pump inhibitors have on the efficacy of immune check point inhibitors in metastatic malignant melanoma? J Clin Oncol 2019;37:e21040.

20. Zhao S, Gao G, Li W, et al. Antibiotics are associated with attenuated efficacy of anti-PD-1/PD-L1 therapies in Chinese patients with advanced non-small cell lung cancer. Lung Cancer 2019;130:10-7.

21. Chalabi M, Cardona A, Nagarkar DR, et al. Efficacy of chemotherapy and atezolizumab in patients with nonsmall-cell lung cancer receiving antibiotics and proton pump inhibitors: pooled post hoc analyses of the OAK and
POPLAR trials. Ann Oncol 2020;31:525-31.

22. Iglesias-Santamaría A. Impact of antibiotic use and other concomitant medications on the efficacy of immune checkpoint inhibitors in patients with advanced cancer. Clin Transl Oncol 2020;22:1481-90.

23. Gopalakrishnan V, Spencer CN, Nezi L, et al. Gut microbiome modulates response to anti-PD-1 immunotherapy in melanoma patients. Science 2018;359:97-103.

24. Katayama Y, Yamada T, Shimamoto T, et al. The role of the gut microbiome on the efficacy of immune checkpoint inhibitors in Japanese responder patients with advanced non-small cell lung cancer. Transl Lung Cancer Res 2019;8:847-53.

25. Jackson MA, Goodrich JK, Maxan ME, et al. Proton pump inhibitors alter the composition of the gut microbiota. Gut 2016;65:749-56.

26. Imhann F, Vich VA, Bonder MJ, et al. The influence of proton pump inhibitors and other commonly used medication on the gut microbiota. Gut Microbes 2017;8:351-8.

27. Pierrard J, Seront E. Impact of the gut microbiome on immune checkpoint inhibitor efficacy-a systematic review. Curr Oncol 2019;26:395-403.

28. Milito AD, Canese R, Marino MC, et al. pH-dependent antitumor activity of proton pump inhibitors against human melanoma is mediated by inhibition of tumor acidity. Int J Cancer 2010;127:207-19.

29. Bellone M, Calcinotto A, Filipazzi P, et al. The acidity of the tumor microenvironment is a mechanism of immune escape that can be overcome by proton pump inhibitors. Oncoimmunology 2013;2:e22058.

30. Wang B, Zhang J, Wang JL, et al. Intermittent high dose proton pump inhibitor enhances the antitumor effects of chemotherapy in metastatic breast cancer. J Exp Clin Cancer Res 2015;34:85-96.

31. Mukherjee S, Ibrahimi S, Khalid B, et al. Do proton pump inhibitors modulate the efficacy of anti-PD-1/PDL1 therapy? A retrospective study. J Oncol Pharm Pract 2019;25:762-4.

(English Language Editor: D. Fitzgerald)

Cite this article as: $\mathrm{Li} \mathrm{C,} \mathrm{Xia} \mathrm{Z,} \mathrm{Li} \mathrm{A,} \mathrm{Meng} \mathrm{J.} \mathrm{The} \mathrm{effect} \mathrm{of}$ proton pump inhibitor uses on outcomes for cancer patients treated with immune checkpoint inhibitors: a meta-analysis. Ann Transl Med 2020;8(24):1655. doi: 10.21037/atm-20-7498 


\section{Full search strategy}

A PubMed search was conducted using the following search strategy:

(("Microbiota" [Mesh] OR "Proton Pump Inhibitors" [Mesh]) OR ("proton pump inhibitor" OR "proton pump inhibitors" OR PPI OR PPIs OR Microbiota OR microbiome) OR (esomeprazole OR omeprazole OR pantoprazole OR lansoprazole OR dexlansoprazole OR tenatoprazole OR Rabeprazole OR Ilaprazole)) AND

((Neoplasms OR neoplasm OR carcinoma OR Cancer OR leukemia OR lymphoma OR melanoma OR malignancy OR malignancies OR tumor OR tumors) OR ("Neoplasms" [Mesh] OR "Carcinoma" [Mesh])) AND

(Immune Check point Inhibitor OR Immune Check point Inhibitors OR immune therapy OR immunotherapy OR cytotoxic T-lymphocyte associated antigen-4 OR CTLA-4 OR programmed cell death protein-1 OR programmed cell death protein OR programmed cell deathLigand 1 OR PD-1 OR OR PD-L1 OR Ipilimumab OR tremelimumab OR nivolumab OR pembrolizumab OR atezolizumab OR durvalumab OR avelumab OR cemiplimab OR toripalimab OR sintilimab OR Camrelizumab OR
SHR-1210 OR Tislelizumab)

An EMBASE search was conducted using the following search strategy:

(('microflora'/exp OR 'proton pump inhibitors'/exp OR 'proton pump inhibitor' OR 'proton pump inhibitors' OR ppi OR ppis OR microbiota OR microbiome) OR (esomeprazole OR omeprazole OR pantoprazole OR lansoprazole OR dexlansoprazole OR tenatoprazole OR rabeprazole OR ilaprazole)) AND

('neoplasm'/exp OR neoplasms OR neoplasm OR carcinoma OR cancer OR leukemia OR lymphoma OR melanoma OR malignancy OR malignancies OR tumor OR tumors) AND

('immune checkpoint inhibitor' OR immunotherapy OR 'cytotoxic t lymphocyte antigen 4' OR 'ctla 4' OR 'programmed cell death protein' OR 'pd 1' OR 'programmed cell death-ligand' OR 'pd 11' OR ipilimumab OR tremelimumab OR nivolumab OR pembrolizumab OR atezolizumab OR durvalumab OR avelumab OR cemiplimab OR toripalimab OR sintilimab OR camrelizumab OR 'shr 1210' OR tislelizumab OR 'immune therapy' OR immunotherapy). 